\title{
Defactinib Hydrochloride
}

National Cancer Institute

\section{Source}

National Cancer Institute. Defactinib Hydrochloride. NCI Thesaurus. Code C128039.

The hydrochloride salt form of defactinib, an orally bioavailable, small-molecule focal adhesion kinase (FAK) inhibitor with potential antiang iogenic and antineoplastic activities. Defactinib inhibits FAK, which may prevent the integ rin-mediated activation of several downstream signal transduction pathways, including those involving RAS/MEK/ERK and PI3K/Akt, thus inhibiting tumor cell migration, proliferation, survival, and tumor angiogenesis. The tyrosine kinase FAK, a signal transducer for integrins, is normally activated by binding to integrins in the extracellular matrix (ECM) but may be upregulated and constitutively activated in various tumor cell types. 